\title{
Estrategias lúdicas en el aprendizaje de la nomenclatura química inorgánica
}

\section{Playful strategies in the learning of inorganic chemical nomenclature}

\author{
Verónica Maila-Álvarez \\ Universidad Central del Ecuador, Quito, Ecuador \\ mvmaila@uce.edu.ec \\ https://orcid.org/0000-0002-4139-7636
}

Helen Figueroa-Cepeda

Universidad Central del Ecuador, Quito, Ecuador

hifigueroa@uce.edu.ec

https://orcid.org/0000-0002-6305-487X

Elizabeth Pérez-Alarcón

Universidad Central del Ecuador, Quito, Ecuador

eyperez@uce.edu.ec

https://orcid.org/0000-0002-7739-5931

Jefferson Cedeño-López

Unidad Educativa Municipal San Francisco de Quito, Guayllabamba, Ecuador

sanfranciscodquito@gmail.com

https://orcid.org/0000-0001-6313-7061

(Recibido: 16/12/2019; Aceptado: 18/12/2019; Versión final recibida: 07/01/2020)

Cita del artículo: Maila-Álvarez, V., Figueroa-Cepeda, H., Pérez-Alarcón, E. y Cedeño-López, J. (2020). Estrategias lúdicas en el aprendizaje de la nomenclatura química inorgánica. Revista Cátedra, 3(1), 59-74.

\section{Resumen}

La implementación de nuevas estrategias y metodologías dentro del proceso de enseñanzaaprendizaje en la educación universitaria surge de la necesidad de ofrecer soluciones a las dificultades de los estudiantes en su quehacer académico. En este contexto, en la Carrera de

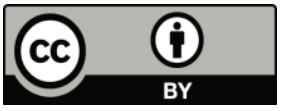


Pedagogía de las Ciencias Experimentales, Química y Biología de la Universidad Central del Ecuador, como unidad académica directamente relacionada en la formación de los nuevos docentes en el área de Química se planteó como objetivo evaluar la incidencia de las estrategias lúdicas en el aprendizaje de la nomenclatura inorgánica. Así, se desarrolló una investigación cuantitativa cuasi experimental aplicada a dos grupos de estudiantes de primer semestre, Grupo Control y Experimental al que se le aplicaron las estrategias lúdicas para el aprendizaje. Se abordaron tres ejes temáticos -Símbolos químicos y valencias; función óxidos: básicos, ácidos, neutros y compuestos y funciones hidróxidos, hidruros y ácidos hidrácidos- y para su valoración se aplicó una evaluación diagnóstica, una al finalizar cada uno de los tres temas y una evaluación sumativa al culminar la unidad. Para el análisis de resultados se trabajó con el 5\% de error. Se aplicaron dos pruebas t de Student que determinan la diferencia significativa entre las medias de dos grupos, y KolmogorovSmirnov, para la distribución de la población, todo procesado con el paquete estadístico SPSS. Kolmogorov mostró que la distribución es normal y la prueba t, que existe diferencia significativa entre los grupos control y experimental en tanto que para las evaluaciones dos y tres, no evidenció diferencia significativa.

\section{Palabras clave}

Aprendizaje, enseñanza, lúdica, nomenclatura, química.

\section{Abstract}

The implementation of new strategies and methodologies in the teaching-learning process in university education arises from the need to respond to the difficulties that students have in their academic work. In this context, in the Career of Pedagogy of Experimental Sciences, Chemistry and Biology of the Central University of Ecuador, in the area of Chemistry was set as an objective to assess the impact of playful strategies on learning inorganic nomenclature. Thus, a quasi-experimental quantitative research applied to two groups of first-semester students, Control and Experimental groups, was used. Three thematic axes were addressed - Chemical symbols and valences; oxides function: acids, neutrals and compounds and hydroxides, hydrides and acids- and five evaluations were applied for titration, one diagnostic, one at the end of each topic and one summative at the end of the unit. 5\% of error was considered for the analysis of the results. Two Student t-tests were applied which determined the significant difference between the means of two groups, and Kolmogorov-Smirnov was employed for the population distribution, all processed with the SPSS statistical package. Kolmogorov showed that the distribution is normal, and the t-test indicated that there is significant difference between the control and experimental groups as for evaluations two and three, it did not show significant difference.

\section{Keywords}

Teaching, learning, play, nomenclature, chamical.

\section{Introducción}

La incorporación de la lúdica en el campo educativo dinamiza el proceso de enseñanzaaprendizaje. Además, facilita la comprensión de temáticas que por su naturaleza son complejas. La nomenclatura química inorgánica es uno de los contenidos que genera dificultad a los estudiantes que se inician en el estudio de la Química, el problema persiste a nivel de educación superior y se evidencia en la Carrera de Pedagogía de las Ciencias Experimentales de la Química y Biología de la Universidad Central del Ecuador. Este inconveniente cobra mayor trascendencia al ser una carrera relacionada con la formación 
docente. Por tal motivo el objetivo del estudio fue evaluar la incidencia de las estrategias lúdicas en el proceso de enseñanza-aprendizaje de la nomenclatura inorgánica.

Frente a la actitud negativa que adoptan los estudiantes al desarrollar los contenidos relacionados con la nomenclatura química inorgánica es imperante incorporar estrategias metodológicas activas. Acciones que promuevan un cambio de actitud y permitan abordar los contenidos de manera participativa. Las estrategias lúdicas se constituyen en una alternativa viable para motivar el estudio de las funciones inorgánicas.

La incorporación de juegos en las actividades de aula reporta importantes beneficios. El juego enmarcado dentro del proceso de enseñanza - aprendizaje despierta el interés. Así lo manifiesta Chacón (2008), "la actividad lúdica es atractiva y motivadora, capta la atención de los alumnos hacia la materia, bien sea para cualquier área que se desee trabajar" (pág. 33). El juego hace que el estudiante se interese por temáticas consideradas como "difíciles" o "áridas", constituyéndose en una estrategia interesante para el docente de ciencias duras. Otro beneficio significativo de las estrategias lúdicas es el desarrollo de habilidades cognitivas como capacidad para resolver problemas, creatividad, toma de decisiones, comunicación asertiva entre otras. Otro aspecto importante que se desarrolla por el juego son las habilidades sociales. Las estrategias lúdicas fortalecen el trabajo cooperativo. Según Martínez et al. (2011), la sinergia entre el juego y el aprendizaje cooperativo permiten "la interacción entre iguales, la aceptación de normas y discusión de ideas, el reconocimiento de los éxitos de los demás y la comprensión de los propios errores" (pág. 403). No cabe duda que la interacción generada a través del juego consolida el aprendizaje, mejora la autoestima y promueve el respeto por los demás. Por lo tanto, son significativos los beneficios de las estrategias lúdicas, vale la pena que el docente las incorpore a su práctica profesional.

Bajo este contexto, en el presente artículo se describen las principales dificultades en el aprendizaje de la nomenclatura química inorgánica, beneficios de la aplicación de las estrategias lúdicas en el proceso de enseñanza-aprendizaje y trabajos relacionados con la lúdica en los diferentes niveles de formación escolar. Se describen los materiales y métodos empleados en la investigación, así como los resultados y conclusiones que se derivan de la experiencia de la incorporación de estrategias lúdicas en la enseñanza-aprendizaje de las funciones químicas inorgánicas en los estudiantes de primer semestre.

\subsection{Dificultades en el aprendizaje de la nomenclatura química inorgánica}

El aprendizaje va más allá de la simple retención de información, implica que el individuo sea capaz de procesar, ubicar bajo un contexto específico y darle un sentido práctico, así lo manifiesta Rivera (2004), "al proceso mediante el cual se construyen las representaciones personales significativas y que poseen sentido de un objeto, situación o representación de la realidad se le conoce como aprendizaje" (pág. 47). Por lo tanto, el aprendizaje comprende procesos cognitivos que permiten vincular lo tangible con lo intangible. Sin embargo, este hecho puede implicar un reto en el aprendizaje de la Química. Nakamatsu (2012) menciona que "el aprendizaje de la Química se complica aún más, pues requiere trabajar a nivel macroscópico (mundo físico) y a nivel sub-microscópico (átomos y moléculas), y utilizar un sistema de representaciones simbólicas (fórmulas, ecuaciones, etc.) y un nuevo lenguaje" (pág. 44). Uno de los contenidos conceptuales de la química inorgánica lo constituye la nomenclatura química. El estudio de este tema ha representado en algunos estudiantes y durante varias generaciones cierto recelo al momento de desarrollarlo. De acuerdo a ciertos autores, entre las razones por las que el estudio de la nomenclatura química infunde dudas en su aprendizaje se menciona: la concepción de difícil comprensión del tema, la aplicación 
de una metodología de enseñanza inadecuada y la memorización como la base de su aprendizaje.

Con respecto a la concepción de la nomenclatura química inorgánica como un tema de difícil comprensión, en el estudio "las TIC en la enseñanza de la Química del ciclo de formación básica para primero, segundo y tercer semestre de la Carrera de Pedagogía de las Ciencias Experimentales, Química y Biología..." realizado por Cedeño (2019), se corroboró la complejidad de la temática antes mencionada. En esta investigación se aplicó una encuesta a los estudiantes de los niveles de formación básica sobre los temas conceptuales que representan mayor dificultad en su aprendizaje dentro de las asignaturas de Química General I, Química General II y Química Inorgánica correspondientes a primer, segundo y tercer semestre respectivamente. Sobre la base de los resultados, el autor señala que para los estudiantes una de las temáticas más complejas lo constituyen las funciones químicas inorgánicas. Así lo señala Cedeño (2019) “... existe mayor dificultad en la unidad cuatro de la asignatura de Química General I, cuyo nombre es Enlaces Químicos e Introducción a la Nomenclatura Química Inorgánica..." (pág. 86).

De las temáticas abordadas dentro de la unidad mencionada (enlaces químicos, generalidades y funciones químicas inorgánicas), tanto las generalidades de la nomenclatura como las principales funciones químicas son para el $25 \%$ y $30 \%$ de estudiantes las temáticas que representan mayor dificultad de compresión. Los porcentajes señalados, si bien no superan el $50 \%$ de estudiantes con similar criterio, corresponden a los de mayor rango, dado que en los temas de la unidad no relacionados con la nomenclatura química no superan el $10 \%$. Es evidente que el estudio de las funciones químicas inorgánicas representa para un porcentaje significativo de estudiantes un verdadero reto al momento de revisarlas.

La metodología de enseñanza de la nomenclatura química es otro aspecto que puede dificultar el aprendizaje de la nomenclatura. Generalmente los docentes en formación de este campo se enmarcan en un modelo de docente espontáneo y los profesionales basan su metodología de enseñanza en técnicas tradicionales. Existe una forma poco adecuada para desarrollar dicho tema por parte de los profesores iniciales. Entre los errores que se cometen de acuerdo a Fernández (2013) están:

... el número de conceptos de índole diversa que son transmitidos simultáneamente. El alumno se ve arrollado por una conjunción de todos ellos que le hacen imposible su correcta asimilación. El perfil disciplinar se encuentra reforzado por el afán clasificatorio, el cual termina introduciendo compuestos como los hidruros metálicos, a igualdad de trato con óxidos o sales...

Es muy común la creencia en el papel primordial de la tabla periódica para saber formular. Es cierto que, entre otras cosas, puede facilitar la retención de valencias/nox y la asimilación de los tipos de compuestos, según los distintos grupos. Pero ello a costa de un esfuerzo memorístico más profundo...

En cuanto al procedimiento utilizado para formular, las reglas "del intercambio" y la "divisibilidad" acarrean inconvenientes nada despreciables. Tal y como se enseñan, aparecen ante el alumno como reglas aritméticas que se aplican mecánicamente, sin mostrar su fundamento químico... (pág. 684-685). 
La forma de enseñanza bajo el modelo espontáneo afecta a los profesores en formación, hecho significativo ya que estos tienden a mantener su práctica profesional en función de su experiencia académica. Es decir, existe la tendencia de replicar los métodos, técnicas y actividades que sus maestros utilizaron con ellos durante su proceso de formación.

Otro elemento que se constituye en un obstáculo para el aprendizaje de la nomenclatura es la memorización. Este fenómeno resulta como consecuencia de la aplicación de estrategias metodológicas de enseñanza tradicionales que fomentan un aprendizaje superficial. Al respecto Castillo et al. (2013) manifiestan que:

El predominio del modelo de enseñanza tradicional en la asignatura de Química, se traduce en un aprendizaje basado sólo en la reproducción de los contenidos dados por el docente, lo cual favorece en los estudiantes la memorización, situación que no se corresponde con lo establecido por la Teoría del Aprendizaje Significativo de David Ausubel propuesta en el año de 1963, quien concibe al estudiante como un procesador activo de la información, debido a que, la transforma y estructura, generándose un aprendizaje significativo, no memorístico (pág. 12).

En el caso de la enseñanza de la nomenclatura química, el empleo de la memorización como principal estrategia de aprendizaje es aún más frecuente. El dominio por parte de los estudiantes de los contenidos conceptuales como símbolos, valencias y fórmulas químicas suelen ser un requisito imprescindible. Esta concepción docente es limitada, pues si bien los símbolos químicos, números de valencia y otros elementos conceptuales requieren de un grado de memorización, este proceso debe estar acompañado principalmente de la comprensión de los mismos y no de la simple repetición.

Por otra parte el uso de recursos bibliográficos inadecuados promueve la repetición de contenidos. De acuerdo a Níaz (2005):

... muchos autores de textos tratan de presentar lo tentativo como definitivo, sin explicar cómo se llegó a determinadas conclusiones bajo ciertas premisas, interpretaciones y evidencias. Así que, en lugar de convencer al estudiante con argumentos, los textos simplemente les presentan la opinión de alguna autoridad científica, para concluir: 'todo el mundo cree que ésta es la verdad'. Ante esta disyuntiva los estudiantes tienen pocas alternativas y en general terminan memorizando el contenido (pág. 411).

Si bien los recursos didácticos juegan un papel significativo en el proceso de enseñanaprendizaje, es necesario realizar una selección cuidadosa de los mismos. Los textos de nomenclatura química son herramientas útiles para la enseñanza siempre que no se limiten a presentar las fórmulas químicas como un simple resultado de la formación de los compuestos. Es necesario el uso de textos que contemplen la explicación de la formación de los compuestos como resultado de las características de los elementos químicos así como del comportamiento de los mismos.

A criterio personal, bajo ninguna concepción debe priorizarse a los contenidos sobre los procesos intelectuales de aprendizaje de los estudiantes. El priorizar los conceptos conllevan a cometer errores a los docentes de química tanto en la ejecución de actividades áulicas como en el uso de recursos didácticos. Estos errores a su vez conducen a un aprendizaje del más bajo nivel como lo es la memorización. 
1.2 Beneficio de la aplicación de las estrategias lúdicas en el proceso de enseñanza-aprendizaje

El juego es una actividad inherente al ser humano, desde temprana edad el juego es parte del desarrollo físico, cognitivo y emocional del mismo. En los primeros años de escolaridad forma parte esencial de las actividades académicas. Por ello ¿por qué no incorporar las estrategias lúdicas en la educación media y superior? En el presente texto se analizarán algunos de los beneficios de las estrategias lúdicas en el proceso de enseñanza-aprendizaje aplicable a los distintos niveles de formación.

Uno de los beneficios más notorios y contundentes de la incorporación del juego a las actividades escolares es sin duda la motivación. Así lo manifiesta Montero (2017), "a partir de la metodología lúdica es que logra el alumno o la alumna iniciar el aprendizaje de una materia, con la misma se pretende incentivar la motivación de continuar con este proceso educativo..." (pág. 76). Sin duda es imprescindible despertar el interés por temáticas que de otro modo serían monótonas o aburridas si se aplican como único recurso metodologías tradicionales. El juego incorporado al proceso educativo puede captar la atención y cambiar la dinámica de la clase al hacerla más activa. La incorporación de otros elementos como las TIC puede constituirse en una metodología efectiva para estudiantes jóvenes y adultos.

El desarrollo de habilidades y destrezas es otro beneficio importante de la incorporación de las actividades lúdicas al proceso de enseñanza-aprendizaje. Son tantas las habilidades y destrezas desarrolladas a través del juego que Chacón (2008) las clasifica en función del área de desarrollo según se representa en la figura 1. Así en el área relacionada con lo físicobiológico, el juego permite la expresión corporal, potencia la rapidez de respuesta ante estímulos como también afina la destreza manual y la coordinación (Chacón, 2008). Si bien es cierto que los beneficios del juego en esta área son sobresalientes en los primeros años de vida del ser humano, en jóvenes y adultos permite mantener e incluso fortalecer su desarrollo físico.
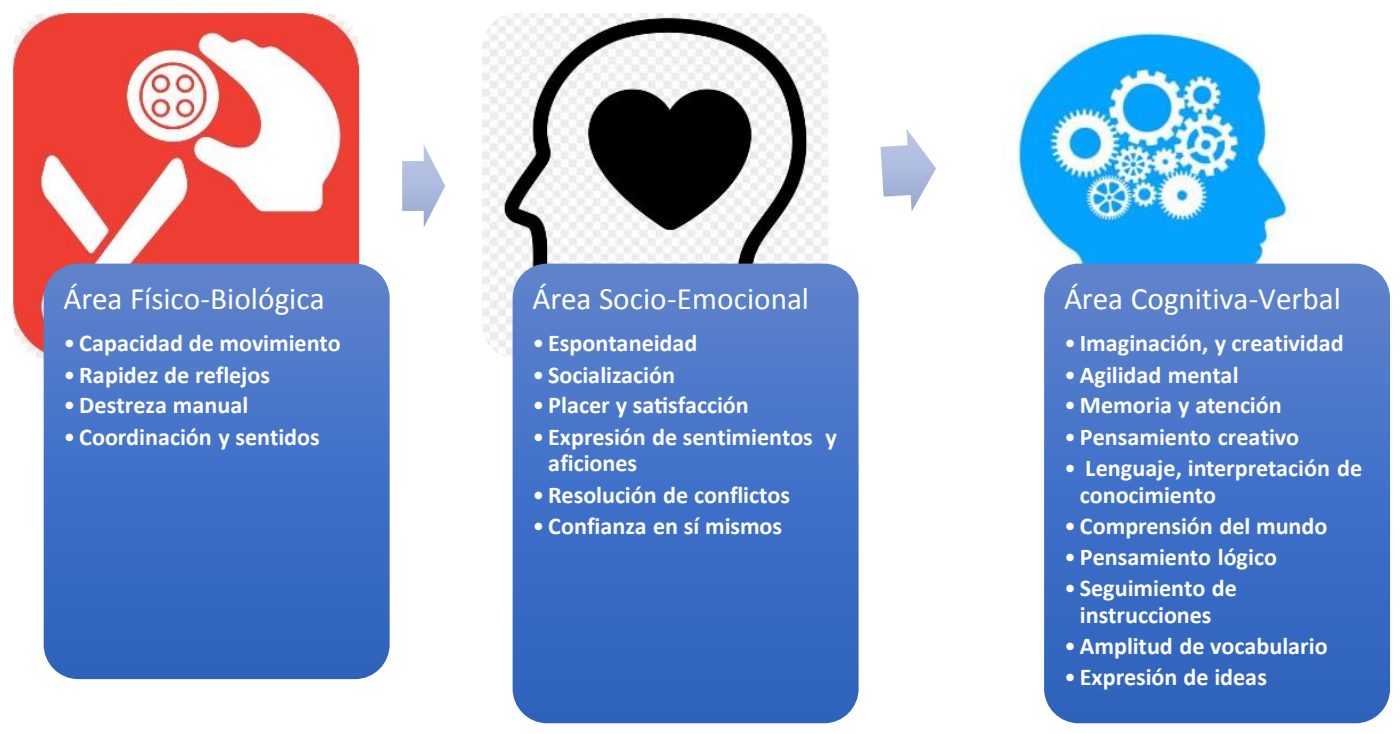

Figura 1. Beneficios de las estrategias lúdicas por áreas del ser humano. Fuente: (Chacón, 2008, pág. 34). 
Otra área importante en la cual contribuye significativamente el juego es el área socioemocional. El ser humano es por naturaleza un ser social, necesita de la interacción positiva con otros individuos. El juego permite mejorar la relación entre docentes y estudiantes e inclusive fortalece la confianza en sí mismos. Este hecho es relevante en estudiantes tímidos que a través de una metodología tradicional su participación es limitada o nula. Así lo manifiesta el equipo Elige Educar (2014) "los alumnos más introvertidos suelen sentirse amenazados cuando están obligados a participar respondiendo una pregunta o resolviendo un ejercicio delante del resto de sus compañeros, lo que genera un bloqueo emocional y la consecuente pérdida en su potencial de aprendizaje..." (pág.1). Las estrategias lúdicas facilitan la integración de todos los estudiantes y por tanto permite mejorar las habilidades sociales de estudiantes introvertidos, ya que el juego mejora la comunicación asertiva y eleva su autoestima.

La lúdica también permite desarrollar el área cognitiva-verbal de los individuos. Las habilidades cognitivas que sobresalen al emplear estrategias lúdicas son la imaginación, la creatividad y el pensamiento lógico. El juego representa un reto que los participantes deben superar, para ello deben vencer dificultades o problemas en los cuales se requiere de agilidad mental, saber seguir instrucciones, concatenar ideas y expresarlas junto al trabajo colaborativo. Oliva (2016) manifiesta que el juego pasa de ser una "simple metodología entretenida y divertida, debido a su dinámica integradora del conocimiento, la gamificación se vuelve un recurso formativo utilizado para acceder a la organización intelectual del individuo, determinando así el funcionamiento cognoscitivo en ciertos mecanismos de cómo aprende el estudiante..." (pág. 44). Por lo tanto, las estrategias lúdicas permiten potenciar las habilidades cognitivas del estudiante facilitando un aprendizaje significativo ya que, gracias a la dinámica del juego, el estudiante enfoca su atención en el mismo, dejando de lado el estrés y la monotonía que tendría una clase tradicional.

\subsection{Trabajo relacionados}

En el proceso de enseñanza-aprendizaje, las estrategias lúdicas han sido aplicadas en los distintos niveles educativos. En torno a lo citado, en la literatura se datan trabajos desarrollados en países latinoamericanos como México, Cuba, Colombia y Argentina, estados en los que el quehacer educativo ha sido fortalecido con la implementación de las estrategias lúdicas a nivel tanto de educación media como superior. Estas experiencias demuestran resultados positivos sin que la edad del estudiante se haya constituido en una limitación. Los trabajos que a continuación se describen brindan una visión más objetiva acerca de lo señalado.

En el año 2014 en Guadalajara Jalisco, México, se publicó el artículo Estrategias didácticas en la enseñanza-aprendizaje: lúdica en el aprendizaje de la nomenclatura química orgánica en alumnos de la escuela preparatoria regional de Atotonilco. En este documento se sintetiza la experiencia de la implementación de los juegos como una estrategia para la enseñanza-aprendizaje. El tema en el que se aplicó la lúdica fue Grupos funcionales correspondiente la Química II del bachillerato General por Competencias. Aquí, los autores manifiestan que es muy importante utilizar el aprendizaje basado en juegos como una estrategia de enseñanza- aprendizaje. Según los autores, la estrategia señalada posibilita a los estudiantes una mayor comprensión y significación de lo aprendido (Zaragoza, et al., 2016, pág. 5).

De igual manera, en el año 2014 en Cuba se divulgó el trabajo de Estrategia didáctica basada en la lúdica para el aprendizaje de la Química en la secundaria básica cubana. En la citada obra, los autores manifiestan que se diseñaron juegos didácticos para la enseñanza de la Química. Como resultados en el grupo experimental se evidenciaron promedios de 
calificaciones mayores que las del curso no experimental acotando que existe aceptación de los juegos por parte de los alumnos. Además, se menciona que la lúdica posibilita el desarrollo del trabajo cooperativo y el incremento del aprendizaje significativo de los estudiantes (Plutin-Pacheco y García-López, 2016, pág. 623).

Más tarde en el año 2018 en Bogotá, Colombia, el estudio Nomenclatura inorgánica: una propuesta lúdica para la enseñanza de la química desarrollada con estudiantes de décimo grado, estuvo encaminado a desarrollar una herramienta lúdica en el décimo curso de la educación media. Este trabajo permitió mostrar la afectación o incidencia del juego en el proceso de aprendizaje. Aquí se señala que, entre otros aspectos, esta estrategia ayuda a lograr una mejor apropiación de la nomenclatura inorgánica tradicional y su relación con el manejo de la fórmula química. En esta investigación se reluce también, el fortalecimiento del trabajo en equipo y el establecimiento de un vínculo entre la construcción y la reconstrucción de las estructuras conceptuales y metodológicas (García, 2018, pág. 45).

En el año 2007 en Cundinamarca, Colombia, ya se publicó la investigación titulada Competencias comunicativas, aprendizaje y enseñanza de las Ciencias Naturales: un enfoque lúdico. En ella su autor determinó que el juego brinda a los estudiantes la posibilidad de desarrollar habilidades comunicativas y construir conocimientos así también que el juego ayuda a fortalecer una competencia académica sana e incrementa la autoestima. Con este enfoque, los estudiantes sienten que ellos ayudan de manera eficaz a construir un verdadero aprendizaje. Por otra parte, el autor señala que la estrategia lúdica estimula un manejo adecuado de la información conceptual y el lenguaje científico. Estos elementos pueden ser construidos, desarrollados y expresados en un contexto grupal de comunicación continua (Palacino, 2007, pág. 295).

De igual manera en el año 1996 en Argentina, en el artículo Cómo favorecer el aprendizaje de la formulación química inorgánica con estrategias no-convencionales, se publicaron los resultados de un estudio basado en la adaptación de un juego para el aprendizaje de la Química. Al respecto, los autores señalan que "las estrategias no-convencionales, como las lúdicas (juegos) convenientemente diseñadas, constituyen un recurso de gran valor que puede aprovecharse como alternativa para lograr que el alumno participe activamente en el proceso de enseñanza-aprendizaje" (pág. 79). Como resultado de este estudio se da a conocer que la ejecución de las actividades lúdicas permitió al grupo en estudio compartir intereses, inquietudes, y responsabilidades, favoreciendo la asimilación y producción de conocimientos (Pandiela, Nuñez, y Macías, 1996, pág. 84).

\section{Métodos y materiales}

La investigación es de tipo cuantitativa y de naturaleza cuasi experimental. Se llevó a cabo en la Carrera de Pedagogía de las Ciencias Experimentales, Química y Biología de la Universidad Central del Ecuador. La población estuvo constituida por estudiantes de primer semestre correspondiente a 35 estudiantes del paralelo B (grupo control) y 43 estudiantes del paralelo $C$ (grupo experimental) al que se le aplicaron estrategias lúdicas para el aprendizaje de la nomenclatura. Por lo tanto, se plantearon las siguientes hipótesis:

$\mathrm{H}_{0}$ : Las estrategias lúdicas no inciden significativamente en el rendimiento académico relacionado con la nomenclatura química inorgánica.

$\mathrm{H}_{1}$ : Las estrategias lúdicas sí inciden significativamente en el rendimiento académico relacionado con la nomenclatura química inorgánica.

La selección de los paralelos se basó en la similitud de condiciones: jornada vespertina de estudio, docente y número de estudiantes. Las actividades lúdicas fueron aplicadas como 
estrategias de refuerzo de los contenidos de la unidad IV correspondiente a las bases de la nomenclatura química inorgánica. Los contenidos se desarrollaron en tres etapas:

- Símbolos químicos y valencias

- Función óxidos; básicos, ácidos, neutros y compuestos

- Funciones hidróxidos, hidruros y ácidos hidrácidos

Al concluir cada etapa se aplicaron juegos grupales sobre la base de dinámicas de competencia y utilizando cuestionarios en línea. Se realizaron 5 evaluaciones; la primera correspondiente al pre test (prueba diagnóstica), tres evaluaciones relacionadas con cada una de las etapas y una evaluación final correspondiente al post test (prueba de unidad).

\section{Resultados y discusión}

En función de los resultados obtenidos con la aplicación del pre test se seleccionó al paralelo C como grupo experimental y al paralelo B como grupo control. El paralelo B obtuvo una $\overline{\mathrm{x}}$ de 8,70 y el paralelo $C$ obtuvo una $\bar{x}$ de 7,03. Al tener el paralelo $C$ la media más baja se quiso verificar el impacto de las estrategias lúdicas en el rendimiento académico.

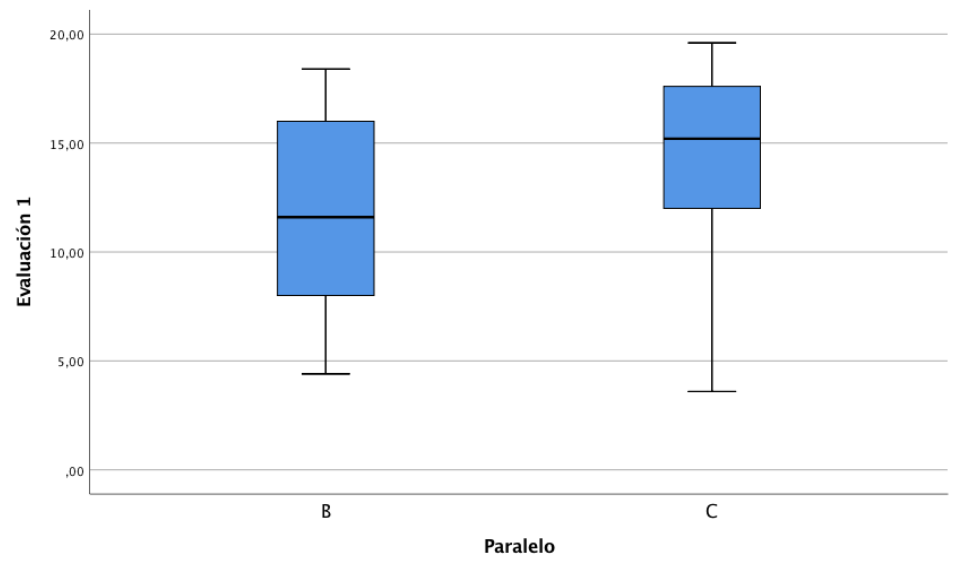

Figura 2. Diagrama de caja y bigotes con $x=12,09$ paralelo $B, x=13,91$ paralelo $C$ evaluación 1 .

Una vez aplicada la prueba de normalidad de Kolmogorov-Smirnov se realizó la prueba $t$ para muestras independientes, se asumieron varianzas iguales para cada uno de las tres evaluaciones. El valor de significancia bilateral para la evaluación 1 es de 0,037. En función que este valor es menor a 0,05 se infiere que hay diferencia significativa entre las calificaciones obtenidas en la evaluación 1 de los grupos control y experimental cuya diferencia de medias se aprecia en la figura 2 grupo control $(11,79)$ y grupo experimental $(14,20)$.

Con respecto a los resultados estadísticos de las evaluaciones 2 y 3 , el valor de significancia bilateral son 0,10 y 0,45 respectivamente. Estos valores superan el 0,05, en consecuencia se deduce que no existe diferencia significativa entre el rendimiento del grupo control y experimental. Pese a que estas diferencias no son estadísticamente significativas, el grupo experimental refleja mejor rendimiento. Ver cuadro 1. Esta diversidad de resultados tiene coherencia con relación a la variable rendimiento académico, ya que esta es de naturaleza multifactorial tal como lo señala Garbanzo (2007):

El rendimiento académico por ser multicausal, envuelve una enorme capacidad explicativa de los distintos factores y espacios temporales que intervienen en el proceso de aprendizaje. Existen diferentes aspectos que 
se asocian al rendimiento académico, entre los que intervienen componentes tanto internos como externos al individuo. Pueden ser de orden social, cognitivo y emocional (pág. 47).

Entre los factores que pudieron haber incidido en el rendimiento académico relacionado con los resultados de las evaluaciones 2 y 3 están vinculados con la inicial familiarización de las normas propias de la nomenclatura, la persistencia del prejuicio sobre el grado de dificultad de la temática junto con problemas del entorno familiar y social que puediese afrontar cada estudiante.

\begin{tabular}{llcr}
\hline Evaluación & \multicolumn{2}{c}{ Grupo control } & \multicolumn{2}{c}{ Grupo experimental } \\
& & $\overline{\mathbf{x}}$ & \\
\hline E. 1 & 11,79 & & 14,20 \\
E. 2 & 12,09 & 13,91 \\
E. 3 & 13,28 & 14,04 \\
\hline
\end{tabular}

Cuadro 1. Medias de las evaluaciones grupo experimental y control 
Con la finalidad de verificar si contribuyó la lúdica a mejorar las condiciones de rendimiento del grupo experimental se aplicó la prueba $t$ correlacional una vez comprobada la normalidad a través de la prueba de Kolmogorov-Smirnov. Los resultados de la misma se pueden apreciar en el cuadro 2 . El valor de significancia bilateral obtenido fue de 0,00 y al ser este valor inferior al 0,05 se evidencia que existe una diferencia significativa entre el pre test y post test. Es decir, se ha pasado de una puntuación de 7,03 en el test inicial a una puntuación de 14,51 en el test final. Al comparar los valores de $t$ calculado $(2,704)$ con $t$ tabular $(-14,202)$ según se muestra en la figura 3 , el valor de $t$ calculado cae por fuera de la zona de aceptación de la hipótesis nula, por lo tanto se rechaza la $\mathrm{H}_{0}$ y se acepta la $\mathrm{H}_{1}$ es decir las estrategias lúdicas sí inciden significativamente en el rendimiento académico relacionado con la nomenclatura química inorgánica.

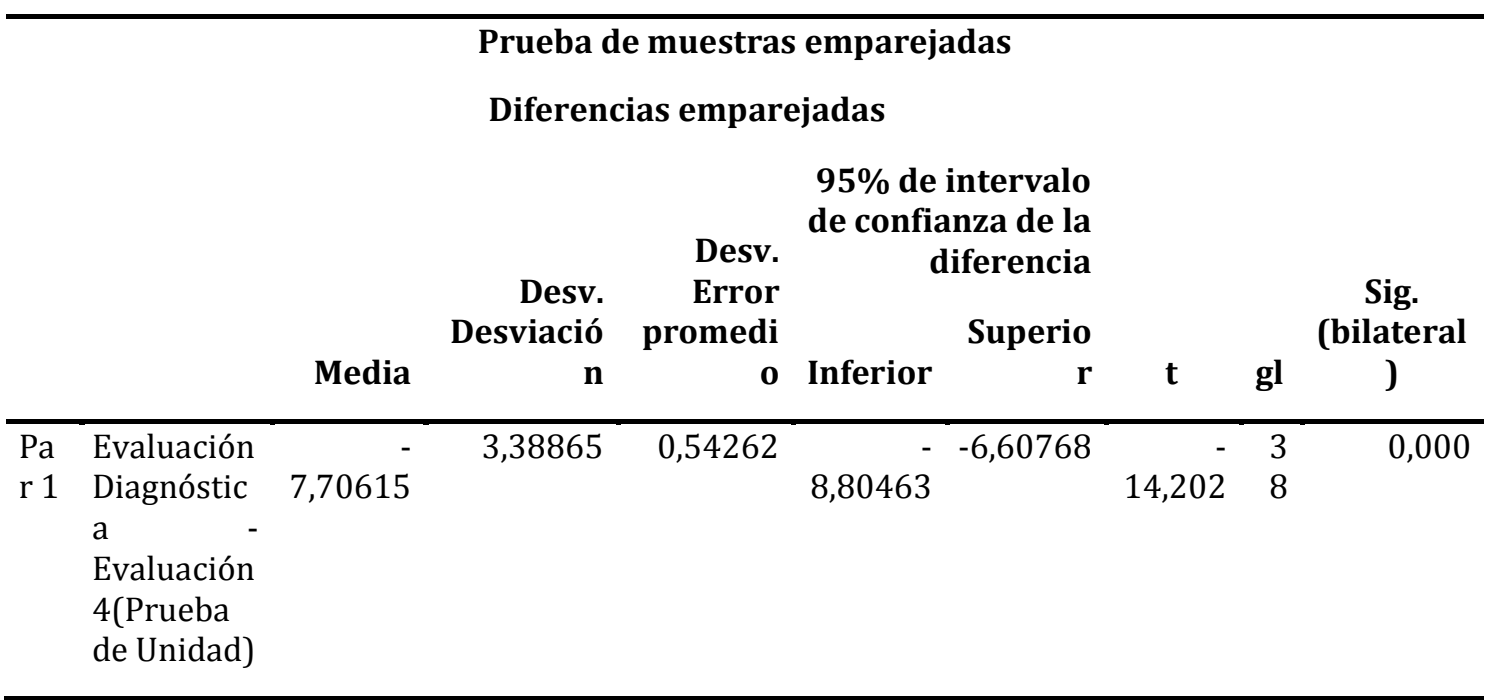

Cuadro 2. Prueba de muestras emparejadas del paralelo C

Nota: Se observa una significancia de 0,000 indicando una diferencia significativa en lo que respecta al inicio del curso y su terminación en cuanto al paralelo C.

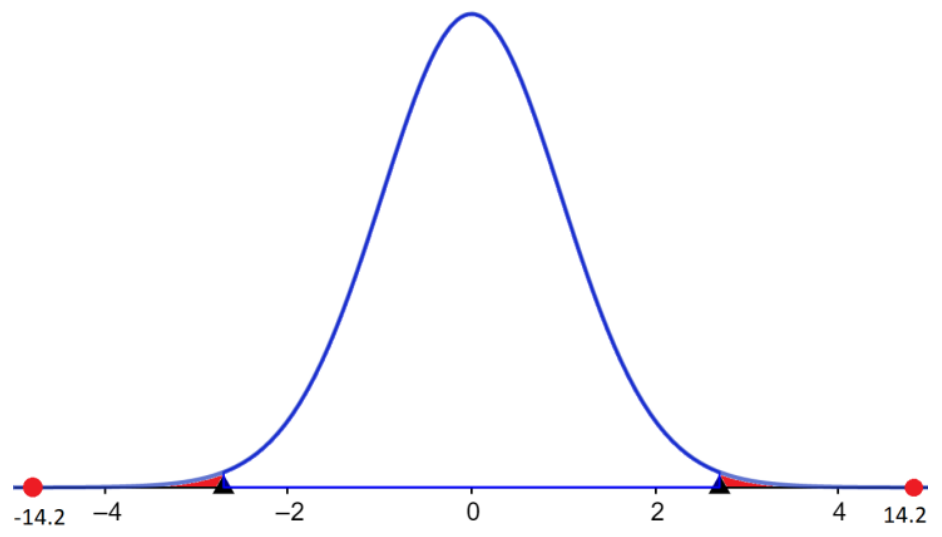

Figura 3. Prueba de hipótesis

Vale la pena señalar que al aplicar la prueba $t$ correlacional al grupo control también se evidencia mejora en el rendimiento académico. Esta diferencia no es tan marcada como en 
el grupo experimental, pues el grupo control aumentó en su media 4,51 puntos mientras que el grupo experimental aumentó 7,48 puntos.

El resultado obtenido entre pre y post test del grupo experimental concuerda con los resultados de estudios similares que aplicaron metodologías lúdicas, así Garza (2014), al implementar el bingo como estrategia metodológica obtuvo un valor de significancia bilateral menor al 0,05 ( $p=0,001)$ y de igual forma Da Silva et al. (2018), al aplicar el juego interactivo Say My Name alcanzó un valor de significancia bilateral menor al 0,05 ( $p=$ 0,0001). En los dos casos se demuestra una diferencia significativa, es decir el grupo experimental tuvo un mejor desempeño que el grupo control.

Es importante resaltar que en la presente investigación el grupo experimental partió en condiciones de rendimiento menor $(x=7,03)$ al del grupo control $(x=8,70)$ y sin embargo en el post test el grupo experimental superó la media del grupo control. Este hecho coincide con la experiencia de Garza (2014), lo que demuestra que las estrategias lúdicas pueden mejorar el rendimiento de grupos aún en condiciones iniciales adversas.

Además de los resultados positivos de carácter cuantitativo se debe resaltar varios aspectos relacionados con la actitud y el comportamiento de los estudiantes durante el desarrollo de las actividades lúdicas. Se observó, por ejemplo, una mejor predisposición durante la clase, dinamismo, compañerismo, alegría, participación, lo cual se puede resumir en una palabra motivación. Frente a las estrategias diferentes a las empleadas normalmente en las clases de nomenclatura química inorgánica, no solo se logró un mejor rendimiento de los estudiantes, sino una actitud de relajamiento, lo cual resulta mucho más beneficioso para el aprendizaje, pues ya no se percibió el temor que sus compañeros de semestres anteriores experimentaron al estudiar nomenclatura química.

\section{Conclusiones}

Se rechaza la $\mathrm{H}_{0}$ y se acepta la $\mathrm{H}_{1}$ que expresa: la incorporación de las estrategias lúdicas al proceso de enseñanza aprendizaje de la nomenclatura química inorgánica permite mejorar el rendimiento académico. Así lo demuestran los resultados de la evaluación 1 al aplicar la prueba $t$ de student para grupos independientes así como los resultados de la aplicación de la prueba $t$ correlacional. La diferencia entre las medias del rendimiento académico en las evaluaciones 2 y 3 sin ser estadísticamente significativas denotan mejor desempeño en el grupo experimental.

Esta experiencia puntual de la aplicación de la lúdica puede proyectarse a otras temáticas que también representan un reto para los estudiantes. Temáticas como, propiedades periódicas, configuración electrónica y cantidades químicas pueden ser desarrolladas mediante las estrategias lúdicas. Los resultados obtenidos en la presente investigación podrían ser mejores con la incorporación de las TIC a las estrategias lúdicas.

La lúdica como estrategia de enseñanza constituye una experiencia positiva aún en personas adultas, ya que genera un ambiente proactivo, promueve la creatividad, el trabajo en equipo y mejora la predisposición hacia temáticas que como la nomenclatura química inorgánica son consideradas áridas. Por lo que, esta estrategia debería incorporarse a la práctica educativa en instituciones de educación superior ya que la edad no es un limitante para desarrollar procesos de aprendizaje a través del juego. 


\section{Bibliografía}

Castillo, A., Ramírez, M. \& González, M. (2013). El aprendizaje significativo de la química: condiciones para lograrlo. Omnia, 19(2), 11-24. Recuperado el 5 de octubre de 2019, de https://www.redalyc.org/pdf/737/73728678002.pdf

Cedeño López, J. (2019). Las TIC en la enseñanza de la Química del ciclo de formación básica para primer, segundo y tercer semestre de la Carrera de Pedagogía de las Ciencias Experimentales, Química y Biología, Facultad de Filosofía, Letras y Ciencias de la Educación, UCE, 2018. Tesis de licenciatura en Ciencias de la Educación, Universidad Central del Ecuador, Quito.

Chacón, P. (2008). El Juego Didáctico como estrategia de enseñanza y aprendizaje ¿Cómo crearlo en el aula? Nueva aula abierta, 5(16), 32-40. Recuperado el 1 de septiembre de 2019, de https://bit.ly/2HkZtTm

Da Silva Júnior, J. N., Nobre, D. J., do Nascimento, R. S., Torres Jr, G. S., Leite Jr, A. J. M., Monteiro, A. J., ... \& Rojo, M. J. (2018). Interactive Computer Game That Engages Students in Reviewing Organic Compound Nomenclature. Journal Chemical Education, 95, 899-902. Doi https://doi.org/10.1021/acs.jchemed.7b00793

Elige Educar (2014). 15 razones por las que los docentes implementan juegos y dinámicas lúdicas en sus clases. Elige Educar. Chile. Recuperado el 9 de octubre de 2019 https://bit.ly/2VAGTza

Fernández González, M. (2013). La formulación química en la formación inicial del profesorado: Concepciones y propuestas. Revista Eureka Sobre Enseñanza Y Divulgación De Las Ciencias, 10, 678-693. Recuperado el 31 de agosto de 2019, de https://revistas.uca.es/index.php/eureka/article/view/2816

Garbanzo Vargas, G. M. (2007). Factores asociados al rendimiento académico en estudiantes universitarios, una reflexión desde la calidad de la educación superior pública. Educación, 31(1) 43-63. Recuperado el 15 de noviembre de 2019, de https://www.redalyc.org/pdf/440/44031103.pdf

García, N. (2018). Nomenclatura inorgánica: una propuesta lúdica para la enseñanza de la química estudiantes de grado décimo del Colegio Agustiniano Ciudad Salitre- CACS. Trabajo de grado para optar por el título de Especialista en Pedagogía. Universitaria Agustiniana Facultad de Humanidades, Ciencias Sociales y Educación, Colombia, Bogotá.

Garza, M. (2014). Impacto de la implementación de una estrategia lúdica para conceptualizar nomenclatura de compuestos orgánicos en estudiantes de educación media superior. Tesis de maestría en Docencia con Orientacion en Educacion Media Superior, México, Monterrey.

Martínez, L., Rincón, E. G., y Domínguez, Á. (2011). El juego y el aprendizaje cooperativo en la enseñanza de las ecuaciones de primer grado. En P.Lestón, (Ed.), Propuestas para la enseñanza de las matemáticas (pp. 397-405). México, DF: Comité Latinoamericano de Matemática Educativa. Recuperado el 1 de septiembre de 2019, de http://funes.uniandes.edu.co/4775/1/MartinezEljuegoALME2011.pdf 
Montero, B. (2017). Aplicación de juegos didácticos como metodología de enseñanza: Una Revisión de la Literatura. Pensamiento Matemático, 7(1), 75-92. Recuperado el 1 de septiembre de 2019, de https://dialnet.unirioja.es/servlet/articulo?codigo=6000065

Nakamatsu, J. (2012). Reflexiones sobre la enseñanza de la química. En Blanco y Negro, 3(2), 38-46. Recuperado el 1 de noviembre de 2019 de https://bit.ly/2Wx65qy

Níaz, M. (2005). ¿Por qué los textos de química general no cambian y siguen una retórica de conclusiones? Educación química, 16(3), 410-415. Recuperado el 31 de agosto de 2019 de DOI: http://dx.doi.org/10.22201/fq.18708404e.2005.3.66104

Oliva, H. A. (2016). La gamificación como estrategia metodológica en el contexto educativo universitario. Realidad y Reflexión, 2016, Año. 16, núm. 44, p. 108-118. Recuperado el 9 de octubre del 2019 de https://bit.ly/313Z5lx

Palacino, F. (2007). Competencias comunicativas, aprendizaje y enseñanza de las Ciencias Naturales: un enfoque lúdico. Revista Electrónica de Enseñanza de las Ciencias., 6(2), 275-298. Recuperado el 10 de octubre de 2019 de https://bit.ly/32aTOtO

Pandiela, P., Macías, A., y Núñez, G. (1997). Cómo favorecer el aprendizaje de la formulación química inorgánica con estrategias no-convencionales. Didáctica de las ciencias experimentales y sociales. (11), 77-84. Recuperado el 2 de septiembre de 2019 de https://core.ac.uk/download/pdf/41560459.pdf

Plutin-Pacheco, N., y García-López, A. (2016). Estrategia didáctica basada en la lúdica para el aprendizaje de la química en la secundaria básica cubana. Revista Cubana de Química, 28(2), 610-624. Recuperado el 2 de septiembre de 2019 de https://bit.ly/2lERxqo

Zaragoza Ramos, E., Torres, O., Mexitli, L., Macías Guzmán, J. O., Núñez Salazar, M. E., Gutiérrez González, R., ... y Gómez Torres, N. A. (2016). Estrategias didácticas en la enseñanza-aprendizaje: lúdica en el estudio de la nomenclatura química orgánica en alumnos de la Escuela Preparatoria Regional de Atotonilco. Educación química, 27(1), 43-51. Recuperado el 2 de septiembre de 2019 de https://bit.ly/2lGL4v5 


\section{Autores}

VERÓNICA MAILA-ÁLVAREZ obtuvo su título de Magíster en Docencia Universitaria y Administración Educativa en la Universidad Tecnológica Indoamérica (Ecuador) en 2011. Obtuvo el título de Especialista en Diseño Curricular por Competencias en la Universidad Tecnológica Indoamérica (Ecuador) en 2010. Obtuvo el título de Doctora en Biología por la Facultad de Filosofía, Letras y Ciencias de la Educación de la Universidad Central del Ecuador en 2001. Obtuvo el título de Licenciada en Ciencias de la Educación, Profesora de Enseñanza Media en la Especialización de Química y Biología por la Facultad de Filosofía, Letras y Ciencias de la Educación de la Universidad Central del Ecuador en 1998.

Actualmente es profesora titular de la Carrera de Pedagogía de las Ciencias Experimentales, Química y Biología de la Facultad de Filosofía, Letras y Ciencias de la Educación de la Universidad Central Ecuador. Ha participado en proyectos de investigación en calidad de Directora e investigadora adjunta, aprobados por la Dirección de Investigación de la Universidad Central del Ecuador. Sus principales temas de investigación se enmarcan en el área de la limnología y en la didáctica de las Ciencias. Es autora de libros y artículos publicados en revistas de Latindex.

HELEN FIGUEROA-CEPEDA obtuvo su título de Magíster en Docencia Universitaria y Administración Educativa en la Universidad Tecnológica Indoamérica (Ecuador) en 2011. Obtuvo el título de Especialista en Diseño Curricular por Competencias en la Universidad Tecnológica Indoamérica (Ecuador) en 2010. Obtuvo el título de Doctora en Biología por la Facultad de Filosofía, Letras y Ciencias de la Educación de la Universidad Central del Ecuador en 2005. Obtuvo el título de Licenciada en Ciencias de la Educación, Profesora de Enseñanza Media en la Especialización de Química y Biología por la Facultad de Filosofía, Letras y Ciencias de la Educación de la Universidad Central del Ecuador en 1997.

Actualmente es profesora titular de la Carrera de Pedagogía de las Ciencias Experimentales, Química y Biología de la Facultad de Filosofía, Letras y Ciencias de la Educación de la Universidad Central Ecuador. Ha participado en proyectos de investigación en calidad de investigadora adjunta, aprobados por la Dirección de Investigación de la Universidad Central del Ecuador. Sus principales temas de investigación se enmarcan en el área de la didáctica de las Ciencias. Es autora de libros y artículos publicados en revistas de Latindex y Open Academic Journal Index.

ELIZABETH PÉREZ-ALARCóN obtuvo su título de Magíster en Docencia Universitaria y Administración Educativa en la Universidad Tecnológica Indoamérica (Ecuador) en 2011. Obtuvo el título de Especialista en Diseño Curricular por Competencias en la Universidad Tecnológica Indoamérica (Ecuador) en 2010. Obtuvo el título de Doctora en Biología por la Facultad de Filosofía, Letras y Ciencias de la Educación de la Universidad Central del Ecuador en 2001. Obtuvo el título de Licenciada en Ciencias de la Educación, Profesora de Enseñanza Media en la Especialización de Química y Biología por la Facultad de Filosofía, Letras y Ciencias de la Educación de la Universidad Central del Ecuador en 1998.

Actualmente es profesora titular de la Carrera de Pedagogía de las Ciencias Experimentales, Química y Biología de la Facultad de Filosofía, Letras y Ciencias de la Educación de la Universidad Central Ecuador. Ha participado en proyectos de investigación en calidad de Directora e investigadora adjunta, aprobados por la Dirección de Investigación de la Universidad Central del Ecuador. Sus principales temas de investigación se enmarcan en el área de la limnología y en la didáctica de las Ciencias. Es autora de libros y artículos publicados en revistas de Latindex. 
JEFFERSON CEDEÑO-LÓPEZ obtuvo su título de Licenciado en Ciencias Naturales y del Ambiente, Biología y Química por la Facultad de Filosofía, Letras y Ciencias de la Educación de la Universidad Central del Ecuador en 2019.

Actualmente se desempeña como docente de enseñanza media en la Unidad Educativa Municipal San Francisco de Quito en Guayllabamba. 